\title{
Rare earth elements: Therapeutic and diagnostic applications in modern medicine
}

\section{EC Giese*}

Center for Mineral Technology, CETEM, Av Pedro Calmon 900, CEP 21941 908, Rio de Janeiro, Brazil

The rare earth elements (REE) are a group of metals comprised of fourteen lanthanide elements [lanthanum (La), cerium (Ce), praseodymium $(\mathrm{Pr})$, neodymium $(\mathrm{Nd})$, promethium $(\mathrm{Pm})$, samarium $(\mathrm{Sm})$, europium $(\mathrm{Eu})$, gadolinium $(\mathrm{Gd})$, terbium $(\mathrm{Tb})$, dysprosium (Dy), holmium (Ho), erbium (Er), thulium (Tm), ytterbium (Yb), lutetium $(\mathrm{Lu})$ ], yttrium $(\mathrm{Y})$ and scandium $(\mathrm{Sc})$ [1]. Their unique physical and chemical properties have rendered them indispensable for a growing number of high-tech technologies as high-performance permanent magnets, magnetic resonance image scanning systems, superconductors and laser technology $[2,3]$.

Lanthanides are also used in many health and medical applications, such as in anti-tumor agent, kidney dialysis medicine and surgical equipment. Due to their optical properties, REE has been used in many imaging techniques such as computed tomography scans, magnetic resonance image (MRI), positron emission tomography (PET) imaging and X-rays [4,5]. The medical applications of REE are summarized in (Table 1).

Gadolinium is the most used REE in medical diagnosis in the MRI. Gd (III) ions enhance MRI images and have also been used in intravenous radio-contrast agents to improve the sensitivity and

Table 1. Medicine applications of rare earth elements

\begin{tabular}{|l|l|l}
\hline REE & Medical applications \\
\hline
\end{tabular}

La Lanthanum oxide nanoparticles can be used for MRI [10]

Ce Cerium-doped lutetium orthosilicate is a scintillator that has been mainly used for

Ce PET imaging, a type of test that reveals tissue and organ function [11]

Pr Praseodymium oxide nanoparticles have been used in radiotherapy techniques [12]

Nd Neodymium has been used in lasers as crystals and is employed in the treatment of

skin cancers, as well as laser hair removal [13]

Am radioisotope $\mathrm{Sm}-153$ has been used to treat severe pain in patients whose tumors

have advanced into bone tissues [14]

Europium presents bioapplications due to its optical properties as nanoprobes with

Eu an emphasis on their heterogeneous/homogeneous biodetection as well as in vitro and in vivo bioimaging [15]

Gd Gadolinium enhances MRI images of tumors, and its magnetic properties are also of use in intravenous radio-contrast agents in MRI scans [6]

Tb A radioisotope Tb-149 has been used in targeted cancer therapy [16]

Dy A radioisotope Dy-165 has been employed in the treatment of rheumatoid knee Dy effusions [17]

Ho Holmium based solid-state lasers have been used for non-invasive medical Ho procedures for treating cancers and kidney stones [18]

Er Erbium-based lasers have been used in medical and dental practice [19]

Tm A radioisotope $\mathrm{Tm}-167$ has been used as power sources in portable X-ray devices

Tm $[20]$

Yb A radioisotope $\mathrm{Yb}-176$ can be used to produce $\mathrm{Lu}-177$ which is known to be a Yb $\begin{aligned} & \text { A romising radioisotope for a medical application [21] } \\ & \text { proming }\end{aligned}$

Lu Lutetium is being researched for its potential uses in targeted radiotherapy, for the Lu advancement of new cancer therapies as prostate cancer [22] specificity of diagnostic images. In this technique, it is possible to visualize the morphology of the body with a very high resolution once Gd (III) ions are the best paramagnetic compounds of the periodic table. The contrast of the images is dependent on magnetic relaxation of the nuclei, and this relaxation can be enhanced by Gd (III) ions, which improves the contrast in magnetic resonance imaging scans with very low toxicity $[6,7]$.

Besides, a considerable variety of luminescent bioassays and sensors also have been developed based on lanthanides that preserve a relatively long-lived emission. Living tissue researchers rely on the Europium for the sensitive luminescence in molecular genetics to mark specific strands of DNA when attached as a tag to complex biochemicals [8]. Nowadays, rare earth elements have also been considered on the anticancer treatment because of their therapeutic radioisotopes, especially as agents in radioimmunotherapy and photodynamic therapy [9]

The use of REE into health and medical applications is now well established. However, much of the future of diagnostic imaging analysis could depend on these paramagnetic elements. Demand for REE is expected to exceed its supply soon. It also considers the possibility of reclaiming the used or worn out REE and reutilizing them; highlighting some companies that have started to recycle the elements, those derived from medical use as well, reducing the demand for newly mined elements. The recycle of REE will be imperative to continue the advancement of RMI and radioisotopes technologies. The future holds many new innovative ideas.

\section{References}

1. Kumari A, Panda R, Jha MK, Kumar JR, Lee JY (2015) Process development to recover rare earth metals from monazite mineral: A review. Minerals Engineering 79: 102-115.

2. Riba JR, López-Torres C, Romeral L, Garcia A (2016) Rare-earth-free propulsion motors for electric vehicles: A technology review. Renewable and Sustainable Energy Reviews 57: 367-379.

3. Cui J, Kramer M, Zhou L, Liu F, Sellmyer D (2018) Current progress and future challenges in rare-earth-free permanent magnets. Acta Materialia 158: 118-137.

4. Townley HE (2013) Applications of the rare earth elements in cancer imaging and therapy. Current Nanoscience.

5. Kostelnik TI, Orbig C (2019) Radioactive main group and rare earth metals for imaging and therapy. Chemical Reviews 119: 902-956.

*Correspondence to: EC Giese, Center for Mineral Technology, CETEM, Av Pedro Calmon 900, CEP 21941 908, Rio de Janeiro, Brazil, E-mail: ellengiese@gmail.com

Received: December 03, 2018; Accepted: December 12, 2018; Published: December 14, 2018 
6. Atar E (2004) Gadolinium-based magnetic resonance imaging contrast agents in interventional radiology. Israel Medical Association Journal 6: 412-414.

7. Mitsumori LM, Bhargava P, Essig M, Maki JH (2014) Magnetic resonance imaging using gadolinium-based contrast agents. Topics in Magnetic Resonance Imaging 23: $51-69$

8. Nishioka T, Yuan J, Yamamoto Y, Sumitomo K, Wang Z, et al. (2006) New luminescent Europium (III) chelates for DNA labeling. Inorganic Chemistry 45: 4088-4096.

9. Kostova I (2005) Lanthanides as Anticancer Agents. Current Medicinal Chemistry Anti-Cancer Agents 5: 591-602.

10. Lanthanum Oxide $\left(\mathrm{La}_{2} \mathrm{O}_{3}\right)$ Nanoparticles - Properties, Applications (2013).

11. Daghighian F, Shenderov P, Pentlow KS, Graham MC, Eshaghian B, et al. (1993) Evaluation of cerium doped lutetium oxyorthosilicate (LSO) scintillation crystal for PET. IEEE Transactions on Nuclear Science 40: 1045.

12. Bakht MK, Sadeghi M, Ahmadi SJ, Sadjadi SS, Tenreiro C (2013) Preparation of radioactive praseodymium oxide as a multifunctional agent in nuclear medicine: expanding the horizons of cancer therapy using nanosized neodymium oxide. Nuclear Medicine Communications 34: 5-12.

13. Littler CM (1999) Hair removal using an Nd: YAG laser system. Hair Restoration and Laser Hair Removal 17: 401-430.

14. Sartor O (2004) Overview of Samarium Sm 153 lexidronam in the treatment of painful metastatic bone disease. Reviews in Urology 6: 3-12. [Crossref]
15. Tu D, Zheng W, Huang P, Chen X (2019) Europium-activated luminescent nanoprobes: From fundamentals to bioapplications. Coordination Chemistry Reviews 378: 104-120.

16. Allen BJ, Blagojevic N (1996) Alpha- and beta-emitting radiolanthanides in targeted cancer therapy: the potential role of terbium-149. Nuclear Medicine Communications 17: 40-47.

17. English RJ, Zalutsky M, Venkatesan P, Sledge CB (1986) Therapeutic application of Dysprosium-165-FHMA in the treatment of rheumatoid knee effusions. Journal of Nuclear Medicine Technology 14: 18-20.

18. Sandhu AS, Srivastava A, Madhusoodanan P, Sinha T, Gupta SK, et al. (2007) Holmium: YAG laser for intracorporeal lithotripsy. Medical Journal Armed Forces India 63: 48-51. [Crossref]

19. Diaci J, Gaspirc B (2012) Comparison of Er: YAG and Er, Cr: YSGG lasers used in dentistry. Journal of the Laser and Health Academy 2012. [Crossref]

20. Scholz KL, Sodd VJ, Blue JW (1976) Production of Thulium- 167 for medical use by irradiation of lutetium, hafnium, tantalum, and tungsten with $590-\mathrm{MeV}$ protons. The International Journal of Applied Radiation and Isotopes 27: 263-266. [Crossref]

21. Park H, Kwon D-H, Cha YH, Kim T-S, Han J, et al. (2008) Stable isotope production of $168 \mathrm{Yb}$ and $176 \mathrm{Yb}$ for industrial and medical applications. Journal of Nuclear Science and Technology 6: 111-116. [Crossref]

22. Emmett L, Willowson K, Violet J, Shin J, Blanksby A, Lee J (2017) Lutetium 177 PSMA radionuclide therapy for men with prostate cancer: a review of the current literature and discussion of practical aspects of therapy. Journal of Medical Radiation Sciences 64: 52-60. [Crossref]

Copyright: (C2018 Jlalia Z. This is an open-access article distributed under the terms of the Creative Commons Attribution License, which permits unrestricted use, distribution, and reproduction in any medium, provided the original author and source are credited. 